POS PROCEEDINGS

\title{
High Energy and Very High Energy Gamma-rays from Galactic Particle Accelerators
}

\author{
Yasunobu Uchiyama* \\ Rikkyo University \\ E-mail: y.uchiyama@rikkyo.ac.jp
}

\begin{abstract}
We review recent observational results on the gamma-ray emission from galactic sources in which efficient particle acceleration can be seen. In particular, we highlight recent progress on our understanding of particle acceleration in supernova remnants, pulsar wind nebulae, and gammaray binaries, based on the results from the Fermi Gamma-ray Space Telescope and ground-based Cherenkov telescopes such as H.E.S.S.
\end{abstract}

Frontiers of Fundamental Physics 14 - FFP14,

15-18 July 2014

Aix Marseille University (AMU) Saint-Charles Campus, Marseille

* Speaker. 